\title{
Ascites and Renal Failure in Liver Disease: Principles of Management
}

\section{Weekly Clinical Conference of the Section of Gastroenterology of the University of Michigan}

\author{
Keith S. Henley, MD
}

Ascites is a common manifestation of chronic liver disease, and it is useful to regard it as evidence of decompensation. An improved understanding of the pathogenesis of ascites has led to significant advances in its therapy. Like any therapy which is apparently effective, this is often applied with enthusiasm which, in turn, can be misguided and, at times, detrimental. For this reason, the prime objective for the treatment of ascites should be clearly understood; it is this objective which has received scant attention in the past.

There is only one major therapeutic benefit to be derived from the treatment of ascites. Relief of ascites by diuresis will lower portal pressure and is, therefore, the best medical means known to us for the prevention of bleeding from esophageal varices (1). Lowering of portal pressure is achieved, to a much lesser extent, by paracentesis. Therefore, the patient whose ascites is difficult, if not impossible, to relieve by the renal route is most likely to suffer a fatal variceal hemorrhage in the hospital. Treatment of ascites per se does not improve liver function, and may even result in liver injury, particularly due to

From the Department of Internal Medicine, Sec tion of Gastroenterology, University of Michigan, Ann Arbor, Mich.

Address for reprint requests: Keith S. Henley, MD, Room 6592, Kresge Research Building 1, Unive sity of Michigan Medical Center, Ann Arbor, Mich 48104. hypokalemia (2). Apart from the important effect on portal pressure, and the cosmetic benefit which is of little clinical importance, the treatment of ascites does little more than demonstrate that the liver is capable of responding to the pharmacologic manipulations employed.

It has been found empirically useful to regard ascites as a special case of the application of Starling's law which was first enunciated in 1896 (3). This law states: "There must, at any time, be a balance between the hydrostatic force of the blood in the capillaries and the osmotic attraction of the blood for the surrounding fluid." This has been given quantitative expression by Atkinson and Losowsky, who found that if the intrasplenic pressure (an index of portal pressure) were plotted against the concentration of serum albumin, almost all patients with ascites fell above a certain line, while patients without ascites fell below that line (3). Again, the line may be expressed empirically by the following ratio:

$$
\frac{10 \times \text { serum albumin }(\mathrm{g} \%)+4}{\text { intrasplenic pressure }\left(\mathrm{cm} \mathrm{H}_{2} \mathrm{O}\right)}
$$

In normal individuals, this ratio is about $4: 5$. If it falls below 1, ascites is likely to occur. Hence, the purpose of therapy is to raise this ratio. This can be done by lowering intrasplenic pressure, by increasing the concentration of albumin in the serum, or 
both. As has already been pointed out, the effective treatment of ascites will, by itself, lower portal pressure; hence, treatment may be self-accelerating and selfperpetuating, and will require modifications in the regimen prescribed.

It is useful to consider separately the denominator and numerator of this ratio. To begin with the denominator, it should be recalled that, in patients with at least a mild or moderate cirrhosis as judged on clinical grounds, bed rest alone will result in a significant fall in portal pressure, and may thereby promote diuresis. This is less evident in patients with cirrhosis in whom the disease is judged to be severe (4). A portal decompression operation, undertaken specifically for relief of ascites, is now hardly ever required, because much of the therapy available can be directed at the numerator.

It is obvious that there is no effective way of enhancing albumin synthesis or of lowering the rate of albumin catabolism or loss. However, in the ratio cited above, albumin is presented as a concentration which is being expressed in $\mathrm{g} / 100 \mathrm{ml}$, and the alternative method to increase its concentration is to remove water. Up to onehalf or more of the total exchangeable albumin in the body may reside in the ascitic fluid, and therefore nothing is gained, and much may be lost, by paracentesis. To increase the concentration of albumin in the serum, then, it is necessary to remove the excess of sodium that goes with it.

It is tempting and a common practice to proceed immediately to a drug regimen. We consider this to be in error because certain fundamentals must be applied to all patients with ascites. The importance of bed rest has already been mentioned. Secondly, by definition, these patients have too much water in their body. For this reason it is futile, and may be dangerous, to attempt a diuretic program without water restriction. Such restriction need not be overly vigorous, and we generally find that patients will tolerate quite well restriction to 1 liter/day. There is no diuresis of any magnitude without natriuresis, and these patients have an excess body sodium just as they have an excess body water (5). Again, it is futile to remove sodium by diuresis without restricting sodium intake. Considering that these patients, untreated, usually excrete either minimal or undetectable amounts of sodium, the so-called "no added salt" diet, which really implies an intake of about 3 or $4 \mathrm{~g}$ sodium/day, is not sufficient. Depending on the degree of ascites, the diet should contain $800-1000 \mathrm{mg}$ sodium. Patients with tense ascites should be given a 450-mg sodium diet, and a skilled dietitian can usually prepare a diet containing only $200 \mathrm{mg}$ sodium. Furthermore, the body potassium is often low. There is really no potassium-sparing diuretic regimen which is also effective. There are only regimens which are more potassium-losing than others (5). Supplementary potassium should be given as potassium chloride, in doses which should be monitored by the serum potassium concentration. It is not unusual to require $120 \mathrm{mEq} /$ day or more. Finally, even with adequate replacement by potassium chloride, hypochloremic alkalosis may occur and can be corrected by administration of lysine monohydrochloride. One ounce of a $20 \%$ solution, given 3 or 4 times a day, will usually restore a chloride deficit. It follows that serum electrolytes should be monitored frequently and that abnormalities should be corrected promptly. It is important to aim at a rate of weight loss which should average between 1 and 2 pounds/day, and treatment 
should be adjusted accordingly. Accurate weighing of such a patient at the same time of day is important.

Having thus established the importance of bed rest, salt and water restriction, and potassium supplementation, one can then proceed to the appropriate drug regimen. Patients with mild to moderate ascites may need no drugs at all, or, if necessary, almost any diuretic drug used alone may suffice.

In patients with moderate to severe ascites, polypharmacy, which has fallen into disrepute elsewhere, is still indicated. This is based on the fact that commonly used diuretic agents act synergistically at different sites in the renal tubule, thereby inhibiting tubular reabsorption of sodium. An aldosterone antagonist, such as spironolactone, while not very effective alone, should be used in conjunction with a carbonic anhydrase inhibitor such as hydrochlorothiazide, or alternatively, with furosemide. The total dose of spironolactone is usually $100 \mathrm{mg} /$ day with hydrochlorothiazide; furosemide is generally given in divided doses totalling about $120 \mathrm{mg} /$ day. These doses should be varied, depending on the patient's clinical response. Should such a program fail to achieve diuresis, the cause for this failure should be investigated. Quite often, it is due to the fact that the patient either does not follow or possibly does not understand the regimen of fluid and salt restriction that has been prescribed. Many patients, particularly alcoholics, are compulsive drinkers, and if they do not receive alcohol, they will drink water. At times, we have found it necessary to institute rigid policing measures, including around-the-clock supervision by a "sitter" and even the turning off of the water supply in the patient's toilet. The best evidence of illict coinsumption of water can be obtained by comparison of intake and output records with the weight chart.
In the truly refractory patient, ethacrynic acid, starting with a dose of about $5 \mathrm{mg}$, which is increased gradually to $50 \mathrm{mg}$ every 12 hours, in addition to the regimen already prescribed, may be effective. Occasionally, as a temporary measure, prednisone in doses of $30 \mathrm{mg} / \mathrm{day}$, by causing inhibition of antidiuretic hormone secretion, may help initiate diuresis (6). Thereafter, it can be withdrawn. If saltpoor human albumin is used it should be given in a minimal volume to maintain fluid restriction. It is not very effective, because it is rapidly catabolized and still contains appreciable quantities of sodium. We have hesitated to use osmotic diuretic agents such as Mannitol because, if they are to be given at all, they must be given rapidly, and there is fear of inducing variceal bleeding if this is done. Given a cooperative patient, conscientious administration of the dietary and drug program, and maintenance of normal electrolyte status, virtually all patients with ascites due to chronic liver disease will respond to this program. Once diuresis has been initiated, it is usually possible to modify the pharmacologic program, to relax the dietary restrictions, and then to monitor the patient's condition and weight when he is being discharged.

A new and more serious situation does arise once the patient presents with severe hyponatremia. This hyponatremia is dilutional-ie, due to the fact that the patient has too much sodium dissolved in far too much water. The temptation to treat this by infusing physiologic or hypertonic saline must be resisted. If there is too much sodium dissolved in too little water, a more rigorous fluid restriction should be imposed, while sodium restriction is maintained. The diuretic regimen may have to be intensified, and as long as the blood urea nitrogen (BUN) and creatinine 
are normal, this condition can be corrected.

A much more ominous situation arises when there is threatened renal failure. This is manifested by a rise in BUN, possibly related to overproduction of urea by the liver in the face of hyponatremia (7), and later, by a rise in serum creatinine, oliguria and eventually anuria. At this state, at least $80 \%$ of the patients will succumb. The mechanism of renal failure is not fully understood, but it appears to be, at least in major part, due to increased vascular resistance of the afferent renal arteriole, and therefore to failure of glomerular perfusion and of glomerular filtration. The tragedy of the situation is that the renal parenchyma per se is unimpaired. This has been proven in man by the successful transplantation of kidneys from patients dying of or with renal failure secondary to cirrhosis. In other words, the renal lesion is reversible, and there is nothing wrong with the kidney per se. It fails because it is joined in circulation to a failing liver. As a corollary, the lesions, if any, found at autopsy in these kidneys, are unimpressive.

In terms of treatment, such patients should be considered to be suffering from acute renal failure, and should be treated conservatively by withdrawal of diuretic and potassium medication, and by strict maintenance of fluid balance. A precipitating or aggravating cause, such as infection, should be sought, and the record should be reviewed for evidence of a prior renal disease. More heroic measures, such as hemodialysis, should be considered only if there is an aggravating factor, such as an operation, which has precipitated this condition. In other words, a patient who has previous, relatively stable, chronic liver disease and whose exacerbation can be traced to an identifiable and correctable cause should be given the benefit of hemodialysis.

Diuretics are often blamed for precipitating renal failure. There is no clear evidence to judge whether or not this is correct. Renal failure secondary to liver disease has been recognized since the nineteenth century, and histologic evidence of nephrotoxicity is almost uniformly absent. However, in weighing risks against benefits, it is clear that excessive zeal in prescribing may do more harm than good.

The rate at which ascitic fluid can be mobilized from the peritoneal compartent is limited (8), and although the plasma volume, in patients with cirrhosis and ascites, is usually elevated, this could be reversed if the rate of diuresis is in excess of the rate at which fluid can be mobilized from the abdomen. As stated in the introductory paragraph, the benefits of treatment of ascites are real, and by judicious management of the patient, these benefits can be achieved with little risk.

\section{REFERENCES}

1. Atkinson $M$ : The effect of diuretics on portal venous pressure. Lancet 2:819-823, 1959

2. Gumucio JJ, Laughrey EG, Henley KS: Effect of hypokalemia on the reductive amination of $\alpha$-ketoglutarate by mitochondria from normal and cirrhotic rat liver. Gastroenterology 56:737-743, 1969

3. Starling EH: On the absorption of fluids from the connective tissue spaces. J Physiol (London) 19:312-326, 1896

4. Leevy CM, Zinke M, Baber J, et al: Observations on the influence of medical therapy on portal hypertension in hepatic cirrhosis. Ann Intern Med 49:837-851, 1958

5. Summerskill WHJ, Clowdus BF II, Rosevear JW: Long-term medical management and complications of "Resistant" ascites. Gut 2:285-296, 1961 
6. Chalmers TC, Morrison RS: Diuretic and steroid therapy in liver disease. Progr Liver Dis 1:338-350, 1961

7. Reissland G: Die Beeinflussung der Harnstoffsynthese der Leber durch Veraenderungen des Natriumchloridgehaltes im Inku- bationsmedium. Klin Wschr 39:445-447, 1961

8. Shear L, Ching S, Gabuzda GJ: Compartmentalization of ascites and edema in patients with hepatic cirrhosis. New Eng J Med 282:1391-1396, 1970 